\title{
COHERENT SHEAVES ON BORDERED RIEMANN SURFACES
}

\author{
BY \\ MARTIN JURCHESCU
}

It is well known that Cartan's Theorems A and B hold for an analytic space $X$ if and only if $X$ is a Stein space [1], [2]. It is also well known that a Riemann surface is a Stein space if and only if it is noncompact.

In this note we consider bordered Riemann surfaces. Let $X$ be a bordered Riemann surface and $B$ the border of $X$. We denote by $\mathcal{O}$ the sheaf of holomorphic functions on the open sets in $X$, and $A \subset \mathcal{O}$ the subsheaf consisting of those holomorphic functions on the open sets $U$ in $X$ which are real-valued on $B \cap U$. Then $\mathcal{O}$ is a sheaf of $\boldsymbol{C}$-algebras, $A$ a sheaf of $\boldsymbol{R}$-algebras, and $A=\mathcal{O}$ if and only if $B=\varnothing$. The elements of $A(U)$ are called analytic functions on $U$, thus distinguishing between holomorphic functions and analytic functions on a bordered Riemann surface. We may consider $X$ as a ringed space either with structure sheaf $\mathcal{O}$ or with structure sheaf $A$. In the first case, using the embedding of $X$ into its double $\hat{X}$ and a theorem of Cartan [1], one easily gets that Cartan's Theorems A and B hold on $X$ if and only if $X$ is noncompact. In this note we shall establish the similar statement for the second case, i.e. when $X$ is considered as a ringed space with structure sheaf $A$. This second case is more natural and more important than the preceding one. We note that $A$ is a coherent sheaf of rings: this fact is an immediate consequence of Oka's theorem.

We begin with an interpretation in terms of sheaves of the symmetry principle We recall that, given a bordered Riemann surface $X$ with border $B \neq \varnothing$, there exists a pair $(\hat{X}, s)$ consisting of a Riemann surface $\hat{X}$ and an antianalytic isomorphism $s: \hat{X} \rightarrow \hat{X}$ (i.e. an analytic isomorphism $s: \hat{X} \rightarrow \hat{X}_{c}$ where $\hat{X}_{c}$ is the conjugate Riemann surface of $\hat{X}$ ) satisfying the following conditions:

(i) $X \subset \hat{X}$ and $\mathcal{O}=\hat{\mathcal{O}} \mid X$, where $\hat{\mathcal{O}}$ is the structure sheaf of $\hat{X}$.

(ii) $s$ is involutive (i.e. $s^{2}=1$ ), $X \cap s(X)=B$ and $s \mid B=1$.

This pair $(\hat{X}, s)$ is unique up to canonical isomorphisms and is called the double of $X$.

For any ringed space $T$, we denote by $\mathrm{Ab}(T)$ the category of sheaves of abelian groups on $T$, Mod $(T)$ the category of modules on $T$ and Coh $(T)$ the category of coherent modules on $T$.

THEOREM 1 (THE SYMMETRY PRINCIPLE). Let $X$ be a bordered Riemann surface with border $B \neq \varnothing$. Consider $X$ as a ringed space with structure sheaf $A$. Then there

Received by the editors June 18, 1968 and, in revised form, March 10, 1969. 
exists a morphism of ringed space (in $R$-algebras)

$$
p=\left(p_{0}, p_{1}\right): \hat{X} \rightarrow X
$$

with the following properties:

(1) The functor $p_{0 *}: \mathrm{Ab}(\hat{X}) \rightarrow \mathrm{Ab}(X)$ is exact, in particular the functor

is exact.

$$
p_{*}: \operatorname{Mod}(\hat{X}) \rightarrow \operatorname{Mod}(X)
$$

(2) For any $\mathscr{G} \in \mathrm{Ab}(\hat{X})$ and any integer $q \geqq 0$, one has $H^{q}(\hat{X}, \mathscr{G})=H^{q}\left(X, p_{0 *}(\mathscr{G})\right)$.

(3) For any $\mathscr{F} \in \mathrm{Coh}(X)$, the canonical morphism $\Theta_{\mathscr{F}}: \mathscr{F} \rightarrow p_{*} p^{*}(\mathscr{F})$ can be embedded in a split exact sequence

$$
0 \longrightarrow \mathscr{F} \stackrel{\Theta_{F}}{\longrightarrow} p_{*} p^{*}(\mathscr{F}) \longrightarrow \mathscr{F} \longrightarrow 0 .
$$

Proof. By definition a morphism of ringed spaces in $R$-algebras $p: \hat{X} \rightarrow X$ is a pair $p=\left(p_{0}, p_{1}\right)$ with $p_{0}$ a continuous map from $\hat{X}$ to $X$ and $p_{1}: A \rightarrow p_{0 *}(\hat{\mathcal{O}})$ a morphism of sheaves on $R$-algebras. We shall define the continuous map $p_{0}: \hat{X} \rightarrow X$ by

$$
\begin{aligned}
p_{0}(x) & =x & & \text { if } x \in X \\
& =s(x) & & \text { if } s(x) \in X
\end{aligned}
$$

and the morphism $p_{1}: A \rightarrow p_{0 *}(\mathcal{O})$ by setting, for any open set $U$ in $X$ and any analytic function $f \in A(U)$

$$
\begin{aligned}
p_{1}(f)(x) & =f(x) & & \text { if } x \in U, \\
& =\overline{f(s(x))} & & \text { if } s(x) \in U .
\end{aligned}
$$

From the classical symmetry principle it follows that $p_{1}(f) \in \mathcal{O}\left(p_{0}^{-1}(U)\right)$ and clearly $p_{1}$ so defined is a morphism of $\boldsymbol{R}$-algebras.

Property (1). If we have an exact sequence

$$
0 \rightarrow \mathscr{G}^{\prime} \rightarrow \mathscr{G} \rightarrow \mathscr{G}^{\prime \prime} \rightarrow 0
$$

in $\mathrm{Ab}(\hat{X})$, then the sequence

$$
0 \rightarrow p_{0 *}\left(\mathscr{G}^{\prime}\right) \rightarrow p_{0 *}(\mathscr{G}) \rightarrow p_{0 *}\left(\mathscr{G}^{\prime \prime}\right) \rightarrow 0
$$

is exact in $\operatorname{Ab}(X)$ because, for any $x \in X$, the stalk at $x$ of this sequence is

if $x \in B$ and

$$
0 \rightarrow \mathscr{G}_{x}^{\prime} \rightarrow \mathscr{G}_{x} \rightarrow \mathscr{G}_{x}^{\prime \prime} \rightarrow 0
$$

$$
0 \rightarrow \mathscr{G}_{x}^{\prime} \times \mathscr{G}_{x}^{\prime} \rightarrow \mathscr{G}_{x} \times \mathscr{G}_{x} \rightarrow \mathscr{G}_{x}^{\prime \prime} \times \mathscr{G}_{x}^{\prime \prime} \rightarrow 0
$$

if $x \notin B$.

Property (2). This is a consequence of the preceding one. In fact, let

$$
0 \rightarrow \mathscr{G} \rightarrow \mathscr{L}^{0} \rightarrow \mathscr{L}^{1} \rightarrow \cdots
$$

be a flask resolution of $\mathscr{G}$, i.e. an exact sequence in the category $\mathrm{Ab}(\hat{X})$ where the sheaves $\mathscr{L}^{i}$ are flask. Since the image by a continuous map of any flask sheaf 
is also a flask one and since the functor $p_{0 *}: \mathrm{Ab}(\hat{X}) \rightarrow \mathrm{Ab}(X)$ is exact, we obtain the flask resolution of $p_{0 *}(\mathscr{G})$ in $\mathrm{Ab}(X)$

etc.

$$
0 \rightarrow p_{0 *}(\mathscr{G}) \rightarrow p_{0 *}\left(\mathscr{L}^{0}\right) \rightarrow p_{0 *}\left(\mathscr{L}^{1}\right) \rightarrow \cdots
$$

Property (3). First we consider the case $\mathscr{F}=A$. Then we have the diagram

$$
A \underset{\rho}{\stackrel{\tau}{\leftrightarrows}} p_{*}(\hat{\mathcal{O}}) \underset{\pi}{\stackrel{\sigma}{\leftrightarrows}} A
$$

where $\rho=p_{1}, \sigma=i \rho$ and where, for any open set $U$ in $X$ and any $g \in \Gamma\left(p_{0}^{-1}(U), \hat{\mathcal{O}}\right)$, we define $\tau(f)$ and $\pi(f)$ by the familiar formulas

$$
\tau(g)(x)=\frac{g(x)+\overline{g(s(x))}}{2}, \quad \pi(g)(x)=\frac{g(x)-\overline{g(s(x)})}{2 i}
$$

for any $x \in U$. We note that the multiplication of $\rho$ by the complex number $i$ is possible because $p_{*}(\hat{\mathcal{O}})$ is a sheaf of $\boldsymbol{C}$-algebras. It is easily seen that $\sigma, \pi, \tau$ are morphisms of $A$-modules when $p_{*}(\hat{\mathcal{O}})$ is considered as an $A$-module by means of $\rho$ and that

$$
\tau \rho=1, \quad \pi \sigma=1, \quad \rho \tau+\sigma \pi=1,
$$

which proves that $p_{*}(\hat{\mathcal{O}}) \approx A \times A$ in the category $\operatorname{Mod}(X)$ with canonical projections $\tau, \pi$ and canonical injections $\rho, \sigma$, hence the sequence

$$
0 \longrightarrow A \stackrel{\rho}{\longrightarrow} p_{*}(\hat{\mathcal{O}}) \stackrel{\pi}{\longrightarrow} A \longrightarrow 0
$$

has the required properties.

Now let $\mathscr{F} \in \operatorname{Coh}(X)$. Then the direct diagram (1) is transformed by the additive functor $\mathscr{G} \leadsto \mathscr{F} \otimes_{A} \mathscr{G}$ into a direct diagram

$$
\mathscr{F} \underset{\rho_{\mathscr{F}}}{\stackrel{\tau_{\mathscr{F}}}{\leftrightarrows}} \mathscr{F} \otimes_{A} p_{*}(\hat{\mathcal{O}}) \underset{\pi_{\mathscr{F}}}{\stackrel{\sigma_{\mathscr{F}}}{\leftrightarrows}} \mathscr{F}
$$

functorially depending on $\mathscr{F}$. Hence

$$
0 \longrightarrow \mathscr{F} \stackrel{\rho_{F}}{\longrightarrow} \mathscr{F} \otimes_{A} p_{*}(\hat{\mathcal{O}}) \stackrel{\pi_{\mathscr{F}}}{\longrightarrow} \mathscr{F} \longrightarrow 0
$$

is a split exact sequence in $\operatorname{Mod}(X)$ and everything is reduced to show that there exists an isomorphism of $A$-modules

$$
\alpha \mathscr{F}: \mathscr{F} \otimes_{A} p_{*}(\hat{\mathcal{O}}) \rightarrow p_{*} p^{*}(\mathscr{F})
$$

satisfying $\alpha \mathscr{F} \rho \mathscr{F}=\Theta_{\mathscr{F}}$. We shall define the $A$-module morphism $\alpha \mathscr{F}$ by

$$
\alpha \mathscr{F}=\Theta_{\mathscr{F}} \tau_{\mathscr{F}}+i \Theta_{\mathscr{F}} \pi_{\mathscr{F}} .
$$

Again the multiplication by $i$ is possible because $p_{*} p^{*}(\mathscr{F})$ is a $p_{*}(\hat{\mathcal{O}})$-module, in particular a sheaf of vector $C$-spaces. Clearly $\alpha \mathscr{F}$ is functorial in $\mathscr{F}$ and we have 
$\alpha \mathscr{F} \rho_{\mathscr{F}}=\Theta_{\mathscr{F}} \tau_{\mathscr{F}} \rho_{\mathscr{F}}+i \Theta_{\mathscr{F}} \pi_{\mathscr{F}} \rho_{\mathscr{F}}=\Theta_{\mathscr{F}}$. It remains to show that $\alpha \mathscr{F}$ is an isomorphism if $\mathscr{F} \in \operatorname{Coh}(X)$. For $\mathscr{F}=A$, we have $\Theta_{\mathscr{F}}=\rho=p_{1}$, hence $\alpha_{\mathscr{F}}=\rho \tau+i \rho \pi=\rho \tau+\sigma \pi=1$, i.e. $\alpha_{\mathscr{F}}$ is an isomorphism for $\mathscr{F}=A$ and by additivity also for $\mathscr{F}=A^{n}$ for any integer $n \geqq 0$. We shall show that $\alpha \mathscr{F}$ is an isomorphism for any $\mathscr{F} \in \operatorname{Coh}(X)$. Since the assertion has a local character on $X$ it will be sufficient to prove it in the case when there exists an exact sequence

$$
A^{p} \rightarrow A^{q} \rightarrow \mathscr{F} \rightarrow 0
$$

in $\operatorname{Mod}(X)$. Since the additive functor $p_{*}: \operatorname{Mod}(\hat{X}) \rightarrow \operatorname{Mod}(X)$ is exact and since the tensor product is a right exact additive functor, functoriality of $\alpha_{\mathscr{F}}$ gives the following commutative diagram with exact rows

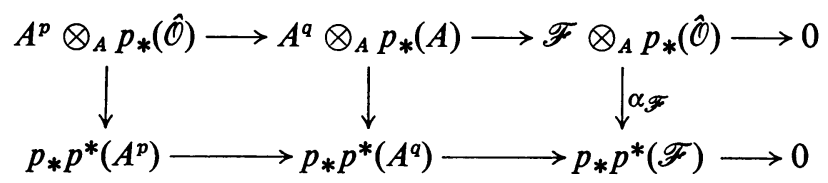

where the first two vertical arrows are isomorphisms. It follows that $\alpha \mathscr{F}$ is also an isomorphism, which completes the proof of the theorem.

THEOREM 2. Let $X$ be a bordered Riemann surface. Consider $X$ as a ringed space with structure sheaf $A$. Then $X$ is noncompact if and only if, for all $\mathscr{F} \in \operatorname{Coh}(X)$, the following two statements hold:

(A) For any $x \in X, \mathscr{F}_{x}$ is generated as an $A_{x}$-module by the global sections of $F$.

(B) $H^{q}(X, \mathscr{F})=0$ for $q \geqq 1$.

Proof. Let $B$ be the border of $X$. The theorem being known in the case $B=\varnothing$, we shall suppose $B \neq \varnothing$. Assume $X$ noncompact and let $\mathscr{F} \in \operatorname{Coh}(X)$.

Assertion (A). From Theorem 1 it follows that we have a commutative diagram with exact rows

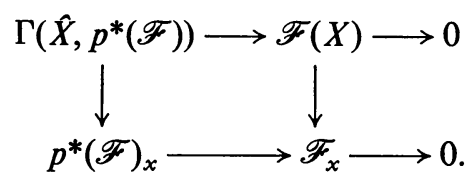

Also $p^{*}(\mathscr{F}) \in \operatorname{Coh}(\hat{X})$ and $(\mathrm{A})$ is true for $\hat{X}$, etc.

Assertion (B). By Theorem 1 we have

$$
H^{q}\left(X, p_{*} p^{*}(\mathscr{F})\right)=H^{q}\left(\hat{X}, p^{*}(\mathscr{F})\right)=0
$$

for $q \geqq 1$ because (B) is true for $\hat{X}$. Furthermore, again by Theorem $1, \mathscr{F}$ is a direct factor of $p_{*} p^{*}(\mathscr{F})$. Hence $H^{q}(X, \mathscr{F})$ is a direct factor of $H^{q}\left(X, p_{*} p^{*}(\mathscr{F})\right)$, and we conclude that $H^{q}(X, \mathscr{F})=0$ for $q \geqq 1$.

Conversely, suppose that (B) is true for every $\mathscr{F} \in \operatorname{Coh}(X)$. By Theorem 1 we have $p_{*} p^{*}(\mathscr{F}) \approx \mathscr{F} \times \mathscr{F}$, hence

$$
H^{q}\left(\hat{X}, p^{*}(\mathscr{F})\right)=H^{q}\left(X, p_{*} p^{*}(\mathscr{F})\right) \approx H^{q}(X, \mathscr{F}) \times H^{q}(X, \mathscr{F})=0 .
$$


It follows that $\hat{X}$ is noncompact, hence $X$ is noncompact.

COROLlaRY 1. Let $X$ be a noncompact bordered Riemann surface and $B$ the border of $X$. For any real-valued analytic function $h$ on $B$, there exists a holomorphic function fon $X$, i.e. a section $f \in \Gamma(X, \mathcal{O})$, such that $\operatorname{Im} f \mid B=h$.

Proof. Let $\mathcal{O}_{B}$ be the restriction of the sheaf $A$ to the border $B$. Then $B$ is a ringed space with structural sheaf $\mathcal{O}_{B}$, more precisely a real analytic manifold of dimension 1. Let $i: B \rightarrow X$ be the inclusion map. Then we have the following exact sequence

$$
0 \longrightarrow A \stackrel{\varepsilon}{\longrightarrow} \mathcal{O} \stackrel{\pi}{\longrightarrow} i\left(\mathcal{O}_{B}\right) \longrightarrow 0
$$

where $\varepsilon$ is the inclusion of $A$ and where, for any open set $U$ in $X$ and any $f \in \Gamma(U, \mathcal{O})$, $\pi_{U}(f)=\operatorname{Im} f \mid U \cap B$. Since $H^{1}(X, A)=0$ by Theorem 1 , the cohomology exact sequence shows that $\pi_{x}: \Gamma(X, \mathcal{O}) \rightarrow \Gamma\left(B, \mathcal{O}_{B}\right)$ is an epimorphism.

COROLlaRY 2. Let $X$ be a noncompact bordered Riemann surface and $B$ the border of $X$. For any real analytic differential form $\beta=h(x) d x$ on $B$, there exists a holomorphic differential form $\omega=f(z) d z$ on $X$ such that $\operatorname{Im} \omega \mid B=\beta$.

(Here $z=x+i y$ is a local coordinate on $X$ and $x$ a local coordinate on $B$.)

Proof. Let $\Omega$ be the sheaf of differential 1-forms on open sets in $X$ having coefficients in $\mathcal{O}$ and let $\Omega^{\prime} \subset \Omega$ be the subsheaf of those differential 1-forms whose coefficients belong to $A$. If $U$ is an open set in $X$, any $\omega \in \Gamma(U, \Omega)$ can be uniquely written as $\omega=\omega^{\prime}+i \omega^{\prime \prime}$ where $\omega^{\prime}$ and $\omega^{\prime \prime}$ are real analytic, and we set $\omega^{\prime}=\operatorname{Re} \omega$, $\omega^{\prime \prime}=\operatorname{Im} \omega$ and $\pi_{U}(\omega)=\omega^{\prime \prime} \mid B \cap U$. Then we have an exact sequence of sheaves of vector $\boldsymbol{R}$-spaces

$$
0 \longrightarrow \Omega^{\prime} \stackrel{\varepsilon}{\longrightarrow} \Omega \stackrel{\pi}{\longrightarrow} i\left(\Omega_{B}\right) \longrightarrow 0
$$

where $\varepsilon$ is the inclusion of $\Omega^{\prime}$ and where $\Omega_{B}$ is the sheaf of differential 1-forms on open sets in $B$ with coefficients in $\mathcal{O}_{B}$. Clearly $\Omega^{\prime}$ is a locally free $A$-module, in particular it is coherent, hence $H^{1}\left(X, \Omega^{\prime}\right)=0$ by Theorem 1 . Again by the exact cohomology sequence we conclude that $\pi_{X}$ is an epimorphism, and the proof is complete.

Let $\mathscr{E}$ be the sheaf of complex valued $C^{\infty}$-functions on open sets in $X$. We denote by $\mathscr{E}^{\prime}$ the subsheaf of $\mathscr{E}$ formed by the $C^{\infty}$-functions $f$ on open sets $U$ in $X$ such that

$$
\frac{\partial^{p} f}{\partial \bar{z}^{p}}=\text { real-valued on } B \cap U
$$

for all integers $p \geqq 0$. If $f \in \mathscr{E}^{\prime}(U)$, then clearly

$$
\frac{\partial^{p+q} f}{\partial \bar{z}^{p} \partial z^{q}}=\text { real-valued on } B \cap U
$$

for all integers $p, q \geqq 0$. We also denote by $\mathscr{E}^{(p, q)}$ the sheaf of differential forms of type $(p, q)$ on open sets in $X$ with coefficients in $\mathscr{E}^{\prime}$. 
COROllary 3. Let $X$ be a noncompact bordered Riemann surface. For any differential form $\alpha=h(z) d \bar{z}$ of type $(0,1)$ on $X$, there exists an $f \in \Gamma\left(X, \mathscr{E}^{\prime}\right)$ such that $\bar{\partial} f=\alpha$ on $X$.

Proof. We consider the sequence

$$
0 \longrightarrow A \longrightarrow \mathscr{E}^{\prime} \stackrel{\bar{\partial}}{\longrightarrow} \mathscr{E}^{\prime(0,1)} \longrightarrow 0
$$

where $\varepsilon$ is the inclusion map. We shall show that this sequence is exact. It is sufficient to prove that, for any rectangle $K$ in the upper half-plane $D=\{z \mid \operatorname{Im} z \geqq 0\}$ with sides parallel to the coordinate axes and any function $\alpha \in \Gamma\left(K, \mathscr{E}^{\prime}\right)$, there is an $f \in \Gamma\left(K^{\circ}, \mathscr{E}^{\prime}\right)$ such that

$$
\frac{\partial f}{\partial \bar{z}}=\alpha \quad \text { on } K^{\circ}
$$

where $K^{\circ}$ is the interior of $K$ in $D$. Let $s: C \rightarrow C$ be the symmetry $s(z)=\bar{z}$. We define the function $\beta$ on $K \cup s(K)$ by

$$
\begin{aligned}
\beta(z) & =\alpha(z) & & \text { if } z \in K \\
& =\overline{\alpha(\bar{z})} & & \text { if } \bar{z} \in K .
\end{aligned}
$$

It is easy to see that $\beta \in \Gamma(K \cup s(K), \mathscr{E})$. We consider the function $f$ on $K \cup s(K)$ defined by

$$
f(z)=\frac{1}{2 \pi i} \iint_{K \cup s(K)} \frac{\beta(\xi)}{\xi-z} d \xi \Lambda d \xi .
$$

One can easily verify that $f(\bar{z})=\overline{f(z)}$, in particular $f$ is real-valued on $K \cap s(K)$. It is known that $\partial f / \partial \bar{z}=\beta$ on the interior of $K \cup s(K)$ in $C$, in particular $\partial f / \partial \bar{z}=\alpha$ on $K^{\circ}$. Since $\alpha \in \Gamma\left(K, \mathscr{E}^{\prime}\right)$, it follows that $f \in \Gamma\left(K^{\circ}, \mathscr{E}^{\prime}\right)$. Because $H^{1}(X, A)=0$, we get by the cohomology exact sequence that the map

is an epimorphism.

$$
\bar{\partial}: \Gamma\left(X, \mathscr{E}^{\prime}\right) \rightarrow \Gamma\left(X, \mathscr{E}^{\prime(0,1)}\right)
$$

REMARK 1. Since the sheaves $\mathscr{E}^{\prime}$ and $\mathscr{E}^{\prime(p, q)}$ are soft, from the preceding corollary it follows that the analog of Dolbeault's theorem holds on bordered Riemann surfaces. We note that on bordered Riemann surfaces one has also an analog of de Rham's theorem. Finally we note that on a bordered Riemann surface $X$ it is important to know the cohomology with coefficients in the sheaf $C^{\prime}$ on $X$ which induces $\boldsymbol{R}$ on $B$ and $C$ on $X-B$. This cohomology can be computed in the general case, using the soft resolution

$$
0 \longrightarrow C^{\prime} \longrightarrow \mathscr{E}^{\prime} \stackrel{d}{\longrightarrow} \mathscr{E}^{\prime(1)} \stackrel{d}{\longrightarrow} \mathscr{E}^{\prime(2)} \longrightarrow 0
$$

(where the notations are clear) and in the case $X$ noncompact the acyclic resolution

$$
0 \longrightarrow C^{\prime} \longrightarrow A \stackrel{d}{\longrightarrow} \Omega^{\prime} \longrightarrow 0 .
$$


REMARK 2. There is a symmetry principle for $C^{\infty}$-functions on a bordered Riemann surface $X$ with a nonempty border $B$. Let $\mathscr{E}$ be the sheaf of complex valued $C^{\infty}$-functions on open sets in $\hat{X}$. Then there exists a morphism of ringed spaces

$$
\pi:(\hat{X}, \hat{\mathscr{E}}) \rightarrow\left(X, \mathscr{E}^{\prime}\right)
$$

(with $\pi_{0}=p_{0}$ ) and for any $\mathscr{E}$-module of finite presentation $\mathscr{F}$, the canonical morphism $\rho_{\mathscr{F}}: \mathscr{F} \rightarrow \pi_{*} \pi^{*}(\mathscr{F})$ can be embedded into a split exact sequence

$$
0 \rightarrow \mathscr{F} \rightarrow \pi_{*} \pi^{*}(\mathscr{F}) \rightarrow \mathscr{F} \rightarrow 0 .
$$

The proof is similar with that one given in the analytic case.

\section{BIBLIOGRAPHY}

1. H. Cartan, Variétés analytiques réelles et variétés analytiques complexes, Bull. Soc. Math. France 85 (1957), 77-99.

2. R. C. Gunning and Hugo Rossi, Analytic functions of several complex variables, PrenticeHall, Englewood Cliffs, N. J., 1965.

3. M. Jurchescu, Bordered Riemann surfaces, Math. Ann. 143 (1961), 264-292.

InSTITUTE OF Mathematics,

BUCHAREST, RUMANIA 